\title{
Results in Precision Multiboson+Jet Phenomenology
}

\section{Francisco Campanario}

Institute for Theoretical Physics, KIT, 76128 Karlsruhe, Germany

\section{Christoph Englert*}

Institut for Particle Physics Phenomenology, Department of Physics,

Durham University, Durham, United Kingdom, and

Institut für Theoretische Physik, Universität Heidelberg, Germany

E-mail: christoph.englertedurham.ac.uk

\section{Michael Rauch}

Institute for Theoretical Physics, KIT, 76128 Karlsruhe, Germany

\section{Michael Spannowsky}

Institut for Particle Physics Phenomenology, Department of Physics, Durham University, Durham, United Kingdom

\section{Dieter Zeppenfeld}

Institute for Theoretical Physics, KIT, 76128 Karlsruhe, Germany

\begin{abstract}
We review recent results in precision multiboson+jet phenomenology at the LHC. We discuss strategies how to compute these processes at NLO QCD and examine the impact of the perturbative corrections on the expected phenomenology, especially in the context of anomalous gauge boson couplings searches.
\end{abstract}

10th International Symposium on Radiative Corrections (Applications of Quantum Field Theory to Phenomenology) - Radcor2011

September 26-30, 2011

Mamallapuram, India

\footnotetext{
* Speaker.
} 


\section{Introduction}

The production of multiple electroweak bosons provides important channels to test LHC data against the well-established Standard Model (SM). As the mechanism of electroweak symmetry breaking is currently unknown, precise predictions of electroweak gauge boson production rates are very important for quantifying deviations from the SM. While the identification of electroweak bosons, especially in their leptonic decay channels, is well established experimentally, the theoretical uncertainties we face at hadron colliders are manifold.

Total rates and differential distributions suffer from severe theoretical ambiguities if they are limited to the leading order approximation in the perturbative series' expansion. The uncertainties intrinsic to fixed order calculations are conventionally assessed by investigating variations of renormalization and factorization scales. The size of these theoretical uncertainties turns out to be particularly large when we deal with a LO color singlet final state configuration. This is realized in pure electroweak production processes such as $p p \rightarrow V V$ or $p p \rightarrow V V V\left(V=W^{ \pm}, Z, \gamma\right)$. In these channels the truncation of the perturbative series at LO means that we force QCD to the odd situation where no color gets transferred from the initial to the final state. In other words, the next-to-leading order QCD corrections to $p p \rightarrow V V, V V V$ [1-3] are large due to kinematically and dynamically unsuppressed initial state parton radiation at large center-of-mass energies. In fact, extra parton emission (i.e. the real emission contribution) is required for the cross sections' infrared safety only in the soft and collinear limit. Hence, a reasonable question to ask is whether we should include hard and resolvable jet emission at all. Vetoing the additional jet activity, i.e. computing the exclusive hadronic cross section to next-to-leading order, has been shown to quickly produce unreliable results [4-7] and should therefore be considered as a path which should be avoided phenomenologically. Even worse from a theoretical point of view, an appropriate choice of the jet veto scale can be utilized to balance the scale dependencies of the (IR-regulated) real emission contribution with the remaining parts of the NLO cross section. For e.g. NLO QCD $W \gamma$ production processes $(\sqrt{s}=14 \mathrm{TeV})$ this is achieved by an experimentally reasonable veto scale choice of $p_{T}^{\text {veto }} \simeq 50 \mathrm{GeV}$.

However, many search strategies for new physics in the context of multiboson production build upon specific final state kinematical configurations and features of the scattering amplitude which are special to the leading order approximation only. An example is the search for anomalous trilinear couplings in $W^{ \pm} \gamma$ production [8] via the identification of the so-called radiation zero [9], which is a coherence effect subject to distortion when additional parton radiation is taken into account [10].

Therefore, two serious limitations are seemingly avoided by imposing a jet veto in anomalous couplings searches: (i) the cross section seems to be perturbatively stable, and (ii), the radiation zero (and the sensitivity to anomalous couplings in total) improves compared to the inclusive NLO computation. However, this comes at the price of an uncertain theoretical prediction which can not be trusted at the currently known order of the perturbative series' expansion.

From this perspective the computation of multiboson+jet cross sections is important for two reasons. On the one hand, adding NLO precision to the one-jet-inclusive production provides an important piece of the full NNLO multiboson production. On the other hand, theoretical uncertainties of the vetoed cross section can be addressed with sufficient precision. As a side benefit we can 

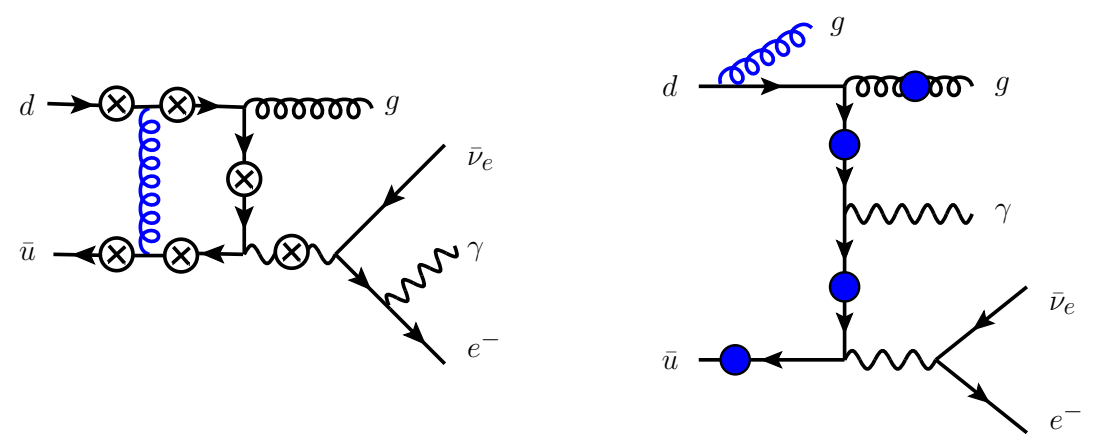

Figure 1: Sample Feynman graphs contributing to $p p \rightarrow W \gamma+$ jet production at NLO QCD. Left: Born and virtual contributions (the crosses mark possible photon couplings). Right: real emission contribution. The $p p \rightarrow W Z+$ jet Feynman graphs follow by replacing the photon with the effective $\left(\gamma^{\star}, Z\right)$ decay current. The figures are taken from Ref. [4].

precisely analyze the potential sensitivity to anomalous couplings which arises from recoils against important initial state radiation.

\section{Inclusive searches for anomalous couplings}

We consider the most general $C$ and $P$ invariant extensions of the electroweak gauge sector [11], which modifies the trilinear $W W \gamma$ vertex

$$
\mathscr{L}_{W W \gamma}=-i e\left[W_{\mu v}^{\dagger} W^{\mu} A^{v}-W_{\mu}^{\dagger} A_{v} W^{\mu v}-\kappa_{\gamma} W_{\mu}^{\dagger} W_{v} F^{\mu v}+\frac{\lambda_{\gamma}}{m_{W}^{2}} W_{\lambda \mu}^{\dagger} W_{v}^{\mu} F^{v \lambda}\right]
$$

and the trilinear $W W Z$ vertex

$$
\mathscr{L}_{W W Z}=-i e \cot \theta_{w}\left[g_{1}^{Z}\left(W_{\mu v}^{\dagger} W^{\mu} Z^{v}-W_{\mu}^{\dagger} Z_{v} W^{\mu v}\right)-\kappa_{Z} W_{\mu}^{\dagger} W_{v} Z^{\mu v}+\frac{\lambda_{Z}}{m_{W}^{2}} W_{\lambda \mu}^{\dagger} W_{v}^{\mu} Z^{v \lambda}\right] .
$$

Unitarity of cross sections at high energy scales requires the anomalous parameters $\left(\kappa_{\gamma}, \kappa_{Z}, \lambda_{\gamma}, \lambda_{Z}, g_{1}^{Z}\right)$ to be understood as formfactors with a momentum dependence such that for large momentum transfers we recover the SM from Eqs. (2.1) and (2.2). Conventional choices are dipole profiles [12], e.g. $\kappa_{\gamma}=1+\Delta \kappa_{0} /\left(1+m_{W \gamma}^{2} / \Lambda^{2}\right)$, where $m_{W \gamma}$ denotes the mass of the invariant $W \gamma$ system and $\Lambda$ relates to the cut-off scale underlying the effective theory of Eq. (2.1). The anomalous couplings modify a subset of Feynman graphs which contribute to $p p \rightarrow \ell^{ \pm} \phi_{T} \gamma+$ jet $+X$ and $p p \rightarrow \ell^{ \pm} \ell^{\prime \pm} \ell^{\prime \mp} \not p_{T} \gamma+$ jet $+X$. The contributing Feynman graphs at NLO QCD are indicated in Fig. 1.

We compute the NLO hadronic cross section by straightforward application of the CataniSeymour dipole subtraction [13]. The loop contributions are evaluated using the Passarino-Veltman scheme up to four-point functions [14] and the Denner-Dittmaier reduction [15] for five point integrals and we perform various cross checks to validate our implementation (for details see Refs. [4-7]). In Fig. 2 we show the total inclusive $p p \rightarrow \ell^{-} \bar{v}_{\ell} \gamma+$ jet cross section for anomalous production. The chosen cuts are adopted from typical searches for anomalous couplings which seek to project out the phase space regions with large momentum transfers in the $W W \gamma(W W Z)$ 

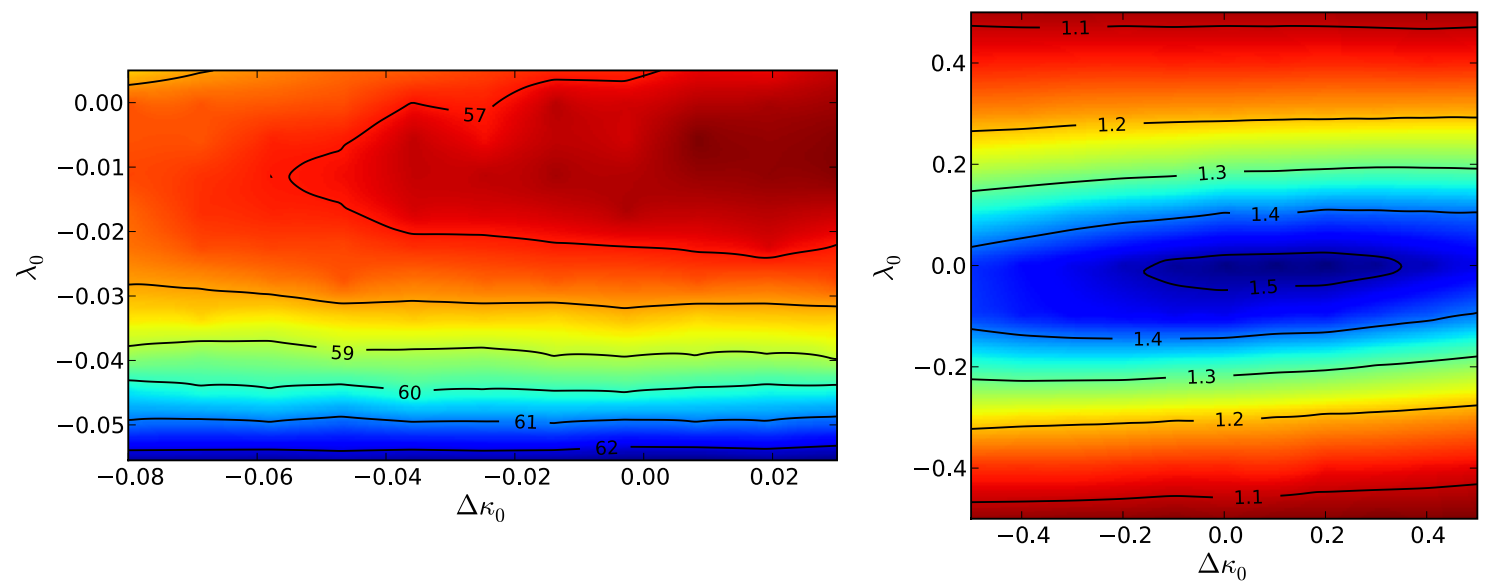

Figure 2: Inclusive NLO QCD $p p \rightarrow \ell^{-} \not p_{T} \gamma+$ jet $+X$ cross section (left, in fb) and K-factor contours (right) at the LHC after applying typical search cuts, as a function of anomalous coupling parameters $[4,12,16]$. The cutoff scale in the dipole form factor is chosen as $\Lambda=2 \mathrm{TeV}$. Figures taken from Ref. [4].

couplings, thus enhancing the sensitivity to anomalous trilinear gauge couplings. In particular, this amounts to hard photons ( $p_{T}^{\gamma} \gtrsim 100 \mathrm{GeV}$ ) or $Z$ s recoiling against the $W$ s (in the following we focus on the $W W \gamma$ vertex [4], for further details on $W W Z$ see Ref. [5]).

The QCD corrections turn out to be most sizable for anomalous couplings choices close to the SM $\left(\lambda_{i}=0, \kappa_{i}=1\right.$. This is a consequence of the differential $K$ factor being large around the $p_{T}^{\gamma}$ threshold while less significant in the distribution's tail. Therefore, large anomalous couplings choices, for which the cross section is significantly enhanced in the $p_{T}$ tails, receive a less sizable relative correction compared to SM-like choices. The photon's transverse momentum distribution, Fig. 3, is typically employed to establish exclusion bounds on anomalous couplings [16]. Even though the (rather) inclusive NLO cross sections' scale dependences of $\sim 10 \%$ turn out to be comparable to the relative difference induced by anomalous couplings ( $c f$. Fig. 2), the distributions

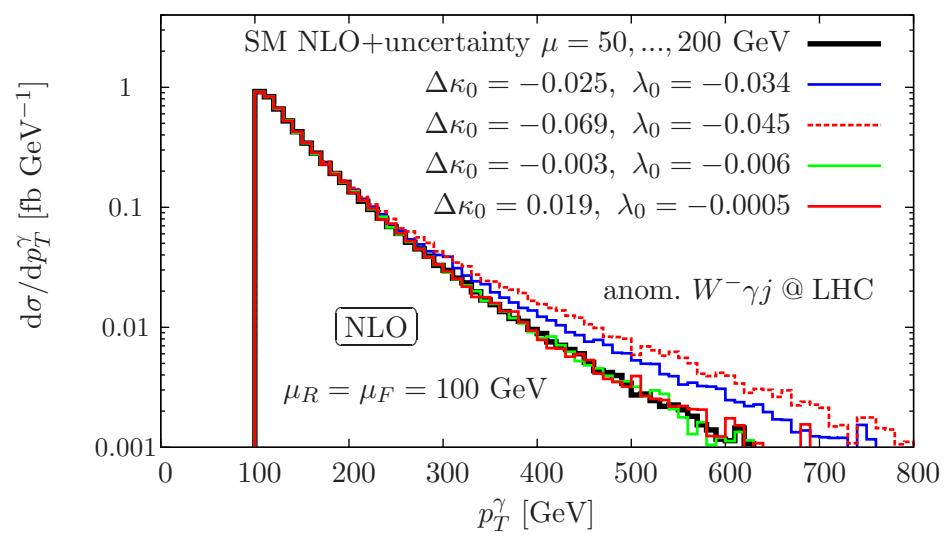

Figure 3: Photon transverse momentum distribution and uncertainty band for SM and anomalous production at NLO QCD from Ref. [4]. Dynamical scale choices have no sizable impact. 
reveal a significant dependence on the anomalous parameters, especially on $\lambda_{i}$ in Eqs. (2.1), (2.2). We perform a binned log-likelihood hypothesis test (taking into account the shape uncertainty that arises from QCD scale uncertainty as a nuisance parameter), $c f$. Ref. [17], to estimate to what extent inclusive searches can help to improve the currently pursued analyses strategies ${ }^{1}$. We find that parameter choices at the edge of the currently allowed LEP bounds on anomalous $W W \gamma$ interactions can be excluded at the $3 \sigma$ level for $\mathscr{L} \simeq 25 \mathrm{fb}^{-1}$ running the $\mathrm{LHC}$ at $14 \mathrm{TeV}$. Note that this result is partly due to the small uncertainty band which follows from the higher NLO precision of the $p_{T}$ distributions.

All results are obtained with an updated version of the VBFNLO package [19], which is publicly available.

\section{3. $W \gamma \gamma+$ jet production at NLO: Toward anomalous quartic couplings}

Among the triple vector boson production channels, $W \gamma \gamma$ has turned out to be of particular interest. It is the channel with the largest $K$ factor $(\sim 4)$ for the integrated cross section, due to the radiation zero, and it is also a very promising channel to measure anomalous quartic couplings [20]. The remaining scale uncertainties at NLO QCD [3] are due to unbalanced gluon-induced real radiation contributions appearing first at NLO, which are computed at LO, e.g., $g q \rightarrow W \gamma \gamma q$. The observation of the radiation zero is also obscured, similar to $W \gamma$ production, by additional real QCD radiation, $W \gamma \gamma+$ jet, as part of the NLO calculation. In Ref. [3] it was shown that an additional jet veto-cut might help in the detection of the radiation zero making visible the dip and also reducing the scale uncertainties for the relevant distributions. However, this procedure raises the question of the reliability of the predictions due to the aforementioned problem with the exclusive vetoed samples. To realistically assess the uncertainties, also concerning anomalous coupling searches, and as an important step towards a NNLO QCD calculation of $W \gamma \gamma$, we have calculated $W \gamma \gamma+$ jet production at NLO QCD. This is the first calculation falling in the category of $V V V+$ jet production and includes the evaluation of the complex hexagon virtual amplitudes, which poses a challenge not only at the level of the analytical calculation, but also on the level of required CPU time computing the full $2 \rightarrow 4$ NLO matrix element.

For the virtual contributions we use the routines computed in Ref. [21] which employ FEYNCAlc [22] and FeynArts [23] in an in-house framework. At the numerical evaluation level, we split the virtual contributions into fermionic loops (Virtual-fermionbox) and bosonic contributions with one (Virtual-box), two (Virtual-pentagons) and three (Virtual-hexagons) electroweak vector bosons attached to the quark line. This procedure allows us to drastically reduce the time spent in evaluating the part containing hexagon diagrams as explained in Refs. [7,21]. The numerical stability of the hexagons' diagram evaluation is discussed in detail in Ref. [21].

\subsection{Results}

For the cross sections and distributions of Tab. 1 and Figs. 4 and 5 we use the CT10 parton distribution set [24] with $\alpha_{s}\left(m_{Z}\right)=0.118$ at NLO, and the CTEQ6L1 set [25] with $\alpha_{s}\left(m_{Z}\right)=0.130$

\footnotetext{
${ }^{1}$ Note that for very hard photons $p_{T}^{\gamma} \geq 100 \mathrm{GeV}$ there is only minimal pollution of misidentified jets and our analysis is essentially background-free [18].
} 

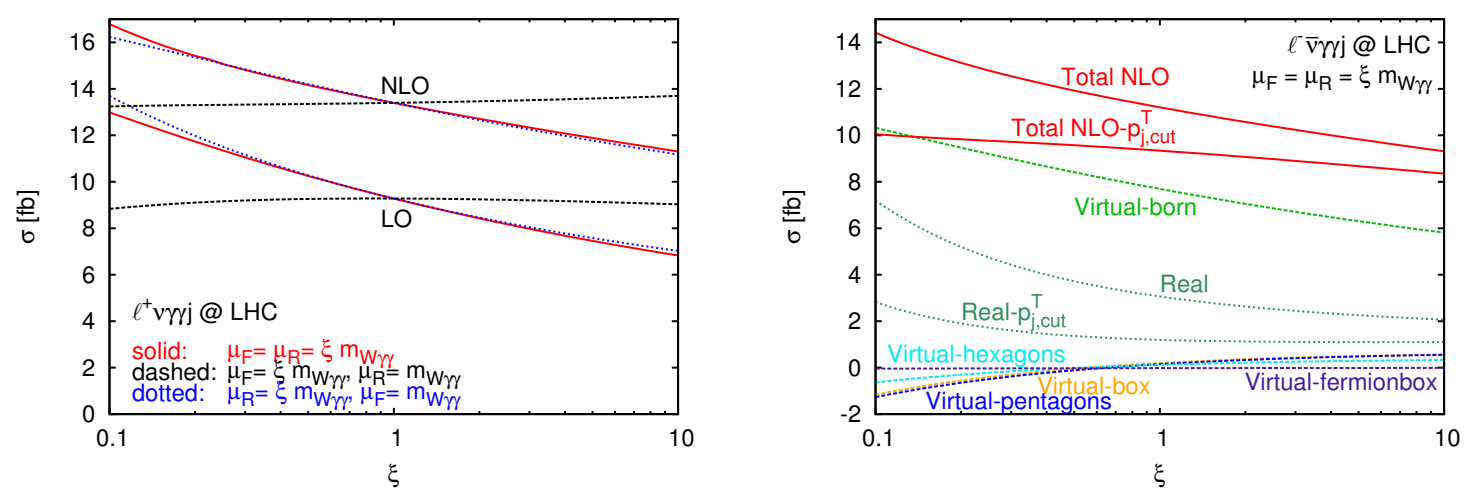

Figure 4: Scale variation of the $\ell^{ \pm} v \gamma \gamma+$ jet production cross sections at the LHC $(\ell=e, \mu)$ taken from Ref. [7]. The cuts are described in the text and we choose $\mu_{R}=\mu_{F}=m_{W \gamma \gamma}$ as central dynamical reference scale. The right panel shows the individual contributions to the NLO cross section according to our classification of topologies. We also show results where we have applied a veto on events with two identified jets having both a transverse momentum larger than $50 \mathrm{GeV}$.

\begin{tabular}{|c|c|c|c|c|}
\hline & $\sigma^{\mathrm{LO}}[\mathrm{fb}]$ & $\sigma^{\mathrm{NLO}}[\mathrm{fb}]$ & $K=\sigma^{\mathrm{NLO}} / \sigma^{\mathrm{LO}}$ & \\
\hline$W^{ \pm} \gamma \gamma+\mathrm{jet}$ & 1.191 & 1.754 & 1.47 & Tevatron \\
\hline$W^{+} \gamma \gamma+$ jet & 4.640 & 6.634 & 1.43 & \multirow{2}{*}{ LHC } \\
\hline$W^{-} \gamma \gamma+$ jet & 3.803 & 5.644 & 1.48 & \\
\hline
\end{tabular}

Table 1: Total LO and NLO cross sections and $K$ factors for $p^{(-)} \rightarrow e^{-} \bar{v}_{e} \gamma \gamma+$ jet $+X$ and $p \stackrel{(-)}{p} \rightarrow$ $e^{+} v_{e} \gamma \gamma+$ jet $+X$ at the Tevatron and at the LHC. Results taken from Ref. [7].

at LO. Further details on the parameter choices can be found in Ref. [7]. Again, we consider $W^{ \pm}$ decays to the first two lepton generations, i.e. the decays $W \rightarrow e v_{e}, \mu v_{\mu}$ and these contributions have been summed in Fig. 5 .

We choose inclusive cuts to study the impact of the inclusive QCD corrections in a general setting: $p_{T}^{j} \geq 20 \mathrm{GeV}, p_{T}^{\ell} \geq 20 \mathrm{GeV}$ (10 GeV at the Tevatron), $p_{T}^{\gamma} \geq 20 \mathrm{GeV}$ (10 GeV at the Tevatron), $\left|\eta_{\ell}\right|,\left|\eta_{\gamma}\right| \leq 2.5$, and an azimuthal angle-pseudorapidity plane separation $R_{\ell \gamma}=\left(\Delta \phi_{\ell \gamma}^{2}+\right.$ $\left.\Delta \eta_{\ell \gamma}^{2}\right)^{1 / 2} \geq 0.4$. For the separation of the charged lepton from observable jets, we choose $R_{\ell j} \geq 0.4$ and we require $R_{\gamma \gamma} \geq 0.4$ for the diphoton separation. Besides the photon-parton isolation criterion according to Ref. [26] with the separation parameter $\delta_{0}=0.7$, we also require a separation between photons and identified jets of $R_{\gamma j} \geq 0.7$.

We compute total $K$ factors of 1.43 (1.48) for $W^{+} \gamma \gamma+$ jet $\left(W^{-} \gamma \gamma+\right.$ jet $)$ production at the LHC, values which are quite typical for multiboson+jet production as found in Refs. [4,5,27]. This moderate $K$ factor (as compared to corrections of $\sim 400 \%$ for $W \gamma \gamma$ production) indicates, as expected, that the $W \gamma \gamma+$ jet production channel is not affected by radiation zero cancellations, since the bulk of the $W \gamma \gamma+$ jet cross section is due to gluon induced processes which do not sport a radiation zero.

The scale dependence of the $W^{+} \gamma \gamma j$ and $W^{-} \gamma \gamma j$ production cross sections turns out to be modest: when comparing $\mu_{R}=\mu_{F}=\xi_{m_{W \gamma \gamma}}$ for $\xi=0.5$ and $\xi=2$, we find differences of $10.8 \%$ 

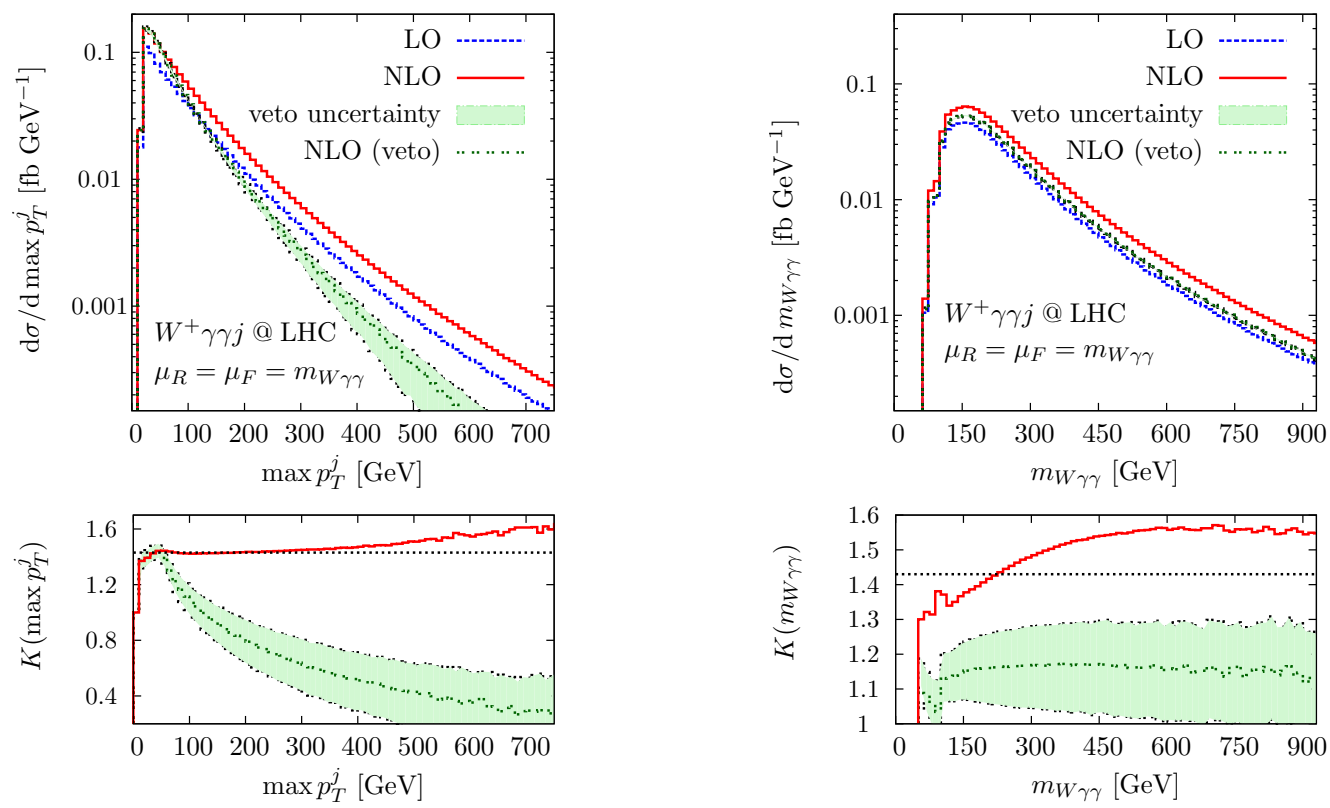

Figure 5: Differential $\max p_{T}^{j}$ and $m_{W \gamma \gamma}$ distribution for inclusive and exclusive $W^{-} \gamma \gamma+$ jet production. Figures taken from Ref. [7].

$(12.0 \%)$, respectively.

The phase space dependence of the QCD corrections is non-trivial and sizable (we again choose $\mu_{R}=\mu_{F}=m_{W \gamma \gamma}$ ). Vetoed real-emission distributions are plagued with large uncertainties - a characteristic trait well-known from $V V+$ jet phenomenology [6,27]. Additional parton emission modifies the transverse momentum and invariant mass spectra among other distributions such as minimum separations, etc. The leading jet becomes slightly harder at NLO as can be inferred from the differential $K$ factor in the bottom panel of Fig. 5. When comparing precisely measured distributions in this channel against LO Monte Carlo predictions, the not-included QCD corrections could be misinterpreted for anomalous electroweak trilinear or quartic couplings $[4,5,8]$ arising from new interactions beyond the SM.

\section{References}

[1] J. Ohnemus, Phys. Rev. D 44 (1991) 1403. J. Ohnemus, Phys. Rev. D 44 (1991) 3477. J. Ohnemus, Phys. Rev. D 47 (1993) 940.

[2] A. Lazopoulos, K. Melnikov and F. Petriello, Phys. Rev. D 76, 014001 (2007). V. Hankele and D. Zeppenfeld, Phys. Lett. B 661, 103 (2008). T. Binoth, G. Ossola, C. G. Papadopoulos and R. Pittau, JHEP 0806, 082 (2008). F. Campanario, V. Hankele, C. Oleari, S. Prestel and D. Zeppenfeld, Phys. Rev. D 78, 094012 (2008). G. Bozzi, F. Campanario, V. Hankele and D. Zeppenfeld, Phys. Rev. D 81, 094030 (2010). G. Bozzi, F. Campanario, M. Rauch, H. Rzehak and D. Zeppenfeld, Phys. Lett. B 696, 380 (2011). U. Baur, D. Wackeroth and M. M. Weber, PoS RADCOR2009, 067 (2010) [arXiv:1001.2688 [hep-ph]]; M. M .Weber, private communication. G. Bozzi, F. Campanario, M. Rauch and D. Zeppenfeld, Phys. Rev. D 84, 074028 (2011).

[3] G. Bozzi, F. Campanario, M. Rauch and D. Zeppenfeld, Phys. Rev. D 83, 114035 (2011). 
[4] F. Campanario, C. Englert and M. Spannowsky, Phys. Rev. D 83 (2011) 074009.

[5] F. Campanario, C. Englert and M. Spannowsky, Phys. Rev. D 82 (2010) 054015.

[6] F. Campanario, C. Englert, S. Kallweit, M. Spannowsky and D. Zeppenfeld, JHEP 1007 (2010) 076.

[7] F. Campanario, C. Englert, M. Rauch and D. Zeppenfeld, Phys. Lett. B 704 (2011) 515.

[8] U. Baur, T. Han and J. Ohnemus, Phys. Rev. D 48 (1993) 5140. J. M. Campbell, R. K. Ellis and C. Williams, JHEP 1107, 018 (2011). For 7 TeV prospects see O. J. P. Eboli, J. Gonzalez-Fraile and M. C. Gonzalez-Garcia, Phys. Lett. B 692, 20 (2010).

[9] R. W. Brown, K. L. Kowalski and S. J. Brodsky, Phys. Rev. D 28 (1983) 624.

[10] F. K. Diakonos, O. Korakianitis, C. G. Papadopoulos, C. Philippides and W. J. Stirling, Phys. Lett. B 303 (1993) 177.

[11] K. Hagiwara, R. D. Peccei, D. Zeppenfeld and K. Hikasa, Nucl. Phys. B 282 (1987) 253.

[12] J. Alcaraz et al. [ALEPH Collaboration and DELPHI Collaboration and L3 Collaboration and ], arXiv:hep-ex/0612034. V. M. Abazov et al. [D $\emptyset$ Collaboration], arXiv:0907.4952. G. Aad et al. [ATLAS Collaboration], arXiv:1111.5570 [hep-ex].

[13] S. Catani and M. H. Seymour, Nucl. Phys. B 485 (1997) 291 [Erratum-ibid. B 510 (1998) 503].

[14] G. Passarino and M. J. G. Veltman, Nucl. Phys. B 160, 151 (1979).

[15] A. Denner and S. Dittmaier, Nucl. Phys. B 658 (2003) 175, Nucl. Phys. B 734 (2006) 62.

[16] M. Dobbs, AIP Conf. Proc. 753, 181 (2005).

[17] A. L. Read, CERN-OPEN-2000-205. A. L. Read, J. Phys. G G28 (2002) 2693-2704. For phenomenological applications see also K. Cranmer and T. Plehn, Eur. Phys. J. C 51 (2007) 415. C. Englert, T. Plehn, P. Schichtel and S. Schumann, Phys. Rev. D 83 (2011) 095009.

[18] M. Escalier, F. Derue, L. Fayard, M. Kado, B. Laforge, C. Reifen, and G. Unal, CERN-ATL-PHYS-PUB-2005-018

[19] K. Arnold et al., Comput. Phys. Commun. 180 (2009) 1661, arXiv:1107.4038 [hep-ph].

[20] S. Godfrey, arXiv:hep-ph/9505252; P. J. Dervan, A. Signer, W. J. Stirling and A. Werthenbach, J. Phys. G 26 (2000) 607 O. J. P. Eboli, M. C. Gonzalez-Garcia, S. M. Lietti and S. F. Novaes, Phys. Rev. D 63 (2001) 075008 P. J. Bell, arXiv:0907.5299 [hep-ph].

[21] F. Campanario, JHEP 1110 (2011) 070.

[22] R. Mertig, M. Bohm and A. Denner, Comput. Phys. Commun. 64 (1991) 345.

[23] T. Hahn, Comput. Phys. Commun. 140 (2001) 418,

[24] H. L. Lai, M. Guzzi, J. Huston, Z. Li, P. M. Nadolsky, J. Pumplin and C. P. Yuan, Phys. Rev. D 82, 074024 (2010)

[25] J. Pumplin, D. R. Stump, J. Huston, H. L. Lai, P. Nadolsky, and W. K. Tung, JHEP 0207 (2002), 012.

[26] S. Frixione, Phys. Lett. B 429 (1998) 369.

[27] V. Del Duca, F. Maltoni, Z. Nagy and Z. Trocsanyi, JHEP 0304 (2003) 059. S. Dittmaier, S. Kallweit and P. Uwer, Phys. Rev. Lett. 100, 062003 (2008). F. Campanario, C. Englert, M. Spannowsky and D. Zeppenfeld, Europhys. Lett. 88 (2009) 11001. S. Dittmaier, S. Kallweit and P. Uwer, Nucl. Phys. B 826 (2010) 18. T. Binoth, T. Gleisberg, S. Karg, N. Kauer and G. Sanguinetti, Phys. Lett. B 683, 154 (2010). 